\title{
Promoting astronomy for the development of society in Nepal
}

\author{
Suresh Bhattarai ${ }^{1}$ and Sudeep Neupane ${ }^{1,2}$ \\ ${ }^{1}$ Nepal Astronomical Society (NASO), P. Box 21229, Kathmandu, Nepal \\ email: chikursharma@gmail.com \\ ${ }^{2}$ Central Department of Physics, Trbhuvan university, \\ Kirtipur, Kathmandu, Nepal \\ email: sudeepneupane@gmail.com
}

\begin{abstract}
Astronomy as one of the oldest sciences has influenced and spurred steady development of society and culture. Inherent superstitious beliefs and their related rituals that have been hampering the progress and prosperity of nations have been dispelled and reduced considerably through the promotion of astronomical activities at all levels of the society. For disseminating basic knowledge and logic of astronomical facts that were deemed important for the development of our society, various programmes have been conducted through mass media. Many talk programmes, seminars and star parties were organised in different places. Our experiences when planning and executing such programmes are summarised and illustrated. The effectiveness of our programmes with the participation of general public is discussed in detail. Positive results of our activities that have contributed towards creation of substantial awareness of astronomy for the development of our society in Nepal are explained.
\end{abstract}

Keywords. Nepal, history of astronomy, Nepalese calendar

\section{Introduction}

Asian civilisation has always become a great matter of interest to the world because of its civilisation through its arts, cultures and religions. Nepal, though a small country in size $\left(147,181 \mathrm{~km}^{2}\right)$ has significant contributions in the development of Asian civilisations especially in the Indian plate (Bharat Barsha). We have traced the history of astronomy and its connection to our civilisation which is more than 2,000 years old through different evidence available till date. Our achievements were first published in Sanskrit which now have become Sanskrit obsolete because of lack of proper updates.

\section{Historical background}

\subsection{Historical works}

The ancient astronomer Maya wrote the Surya Siddhanta (about 3,000 BCE) which was based on the geocentric theory. The Nepalese calendar is based on this publication. We have stone Hinges in Tehrathun and Memeng in Pachthar, in the eastern part of Nepal, and Umbrellas in the temples of Asham, western part of Nepal, probably 2,000 years old which were used for time keeping purposes and astronomical observations. Other publications before the unification of Nepal in 1793 were Sumati Tantram (576880), Sumati Siddhanta (1409), Bhaswati Baal Bhodhini Tika (1494) by Balbhadra of Jumla, Bhaswati Tika (1514) by Ratna Dev. Gaureshwor Joshi wrote three books on Mathematical Astronomy (1663) which were taken as the significant contributions in the history of Nepalese astronomy. All the publications were written in Sanskrit except 
Sumati Siddhanta which was written in Newari. All the above publications were based on the geocentric theory.

Bhaswati Ko Nepali Tika (1793) by Laxmi Pati Pandey was the first publication written in the Nepali Language. He also built a sundial which is no longer available. In 1953, for the first time, Naya Raj Pant, the famous astronomer of Nepal, wrote Jyotisha (Astronomy) for children. Other publications were: Bigyan Ka Kura (1961 A.D.) by Prof. Dilli Raj Upreti, Antariksha (The Earth) (1964); A general introduction to Space, Universe Planets Constellation, by Bal Chandra Sharma with the assistance of UNESCO, Brahmanda Ra Saur Mandal (1966) by Motikaji Sthapit, its revised and updated edition in 2001; Saur Jagat (2005) by Punya Prasad Oli. These publications were based on the heliocentric theory except Bhaswati ko Nepal Tika.

\subsection{Education}

If we look for the education pattern in astronomy in Nepal, introductory astronomy is included in the Lower Secondary and Secondary level curriculums under Geology and Astronomy which covers $5 \%$ of the total course and in the Higher Secondary level curriculum under a chapter called Universe which gives the introduction to the constituents of universe, planets, stars, galaxies, energy sources of stars, stellar evolution, large scale structure and evolution of universe. But the Proficiency Certificate Level has not included astronomy in its syllabus. Students have no opportunities to study Astronomy/Astrophysics/Cosmology at the undergraduate level in Nepal. Tribhuvan University, the first University of Nepal, had offered the Mathematical Astronomy under B.A. (honors) during 1959-1971 but was later excluded from the curriculum arguing that astronomy was outdated. Some professors advocated the inclusion of astronomy in Geography and Physics but their efforts did not yield fruitful results.

There is no separate department or Institute for astronomy and astrophysics in Nepal. Tribhuvan University is only the university offering Physics at the Master Degree and has offered Gravitation and Cosmology as an elective course at the second year level since 1999. It was in 2005 when Tribhuvan University offered a diploma, Dissertation for Astrophysics and Cosmology. So far, 19 students have completed their Masters thesis in A\&A. Out of which, 7 papers have been published ( 2 in MNRAS, 3 in A\&A and 2 in Ap\&SS, 2 manuscripts are in the process of publication).

\subsection{Institutions}

The Nepal Academy of Science and Technology (NAST) is an independent apex body established in 1982 to promote science and technology in the country $\dagger$. It undertakes studies/reports on the achievements of S\&T in the country. It advises the Government on formulation technology transfer policy and its implementation. It has already organised five Science Congresses in 1988, 1992, 2004 and 2008. It has included outreach as an important tool to communicate about the scientific developments and their applications for the development.

The Government of Nepal has established the B.P. Koirala Memorial Planetarium, Observatory and Science Museum Development Board in 1992 in order to establish Planetaria, Observatories and Science Museums in Nepal and conduct research activities in the area of Astronomy, Astrophysics and Cosmology $\ddagger$. The observatory is in operation since 2008 but the Planetarium and Science Museum are still under construction. It has been providing grants to research scholars and access to the newly constructed building

$\dagger$ see www.nast.org.np

$\ddagger$ see www.planeta-observatory.gov.np 
for their research. It has already organised five Schools and one International School on A\&A in Nepal.

Even though amateur astronomers are always a great asset for astronomy development and outreach activities play a vital rôle to make people aware of astronomy and its consequences, the Government has so far ignored amateur communities and the strength and importance of outreach.

\subsection{Observations}

The history of observational astronomy in Nepal extends more than 2000 years, but telescopic observations are in their infancy. In 1986, Nepal constructed its first observatory -with a telescope donated by the Japanese Government- in the premises of the Balmikee Campus of Nepal Sanskrit University, Kathmandu. The setup stopped working after a year of its establishment. Then NAST got another telescope for observational astronomy but it has never been used. By 2008 the National Observatory started its operations with a 16 inch refractor which now has become the only observatory of Nepal. Amateur astronomical communities have been slowly developing in the nation, and act strongly to reduce inherent superstitious beliefs and their related rituals in our society, which hamper the progress and prosperity of our nation. In the promotion of astronomy is important to dispel and reduce the superstitious beliefs and to lead our nation towards the development of science, society and culture.

\section{Methods}

We have carried out different activities throughout the nation to disseminate astronomical facts among the Nepalese by outreach activities, interactions programs and regular broadcasting and telecasting. We have reached almost every corner of the nation through different FM stations and TV channels and by giving press releases on different astronomical events such as solar and lunar eclipses, meteor showers, the discovery of new planets, the maintenance of the Hubble Space Telescope, etc. During 2007-08, we issued 18 press releases, conducted 9 star parties, 5 interaction programmes among which two with International Delegates (Prof. Chun Lee Kim of Chun Buk National University of South Korea and IAU delegates) and 3 talk programmes (one by Prof. Michele Gerbaldi of IAU), participated in two International events (International Workshop on IHY and Basic Space Science, Bulgaria, 2008 and International Workshop on Zameen Aasmaan : Earth and the Sky, Astrophotography and Image Processing, October 2008, India), published two articles every month on The Rising Nepal, the National English Daily, and published 2,000 copies of solar posters.

\section{Results}

With the above activities we reached every corner of Nepal. People showed interest in astronomy and tried to contact us whenever they saw something strange in the sky and asked questions related to astronomy. We were interviewed for different Television Channels, FM stations, Nepali and English national dailies and weeklies frequently. Media showed keen interest on S\&T and started writing more on S\&T including astronomy.

We found an increasing trend of participants in the observational activities. We found that people were aware of the different astronomical happenings like solar eclipses, lunar eclipses, and meter showers, etc. and their consequences. Our activities developed the scientific temper, humanism and spirit of inquiry and reform among students, working 
professionals and general masses. It made people, especially students, think logically and reasonably for the development of their communities. We found our activities as a key rôle for popularising science among the students. We found that the level of superstitious beliefs had been reduced to some extent in the society of Nepal. Also, we found our activities as an encouragement for the people to study and do research activities.

\section{Discussion}

Astronomical outreach activities among the students and general public have played a significant rôle to popularise all the sciences and hence help to develop technically civilised scientific societies. The construction of Public Astronomical Observatories in different places and the establishment of an Amateur Astronomy Centre for all amateur astronomers should be done to increase the level of astronomy in the nation. As we have seen the significant effort of media to disseminate information regarding astronomical happenings and their consequences, further collaboration with the media should be done for outreach activities to create a scientifically-aware society. We can also work in collaboration with NGOs and INGOs working in the field of astronomy.

Moreover, regarding our geographical distribution, clear and dark skies, we can develop the basis for astro-tourism in the country which will help to increase living standards of the people from rural areas. Also, Nepal can be made a dark sky destination and use its high altitude for some serious research activities establishing research centers in the rain shaded regions like Manang and Mustang.

The introduction of an astronomy syllabus at all the levels of education with proper management of observations will result the development of more scientific communities and increase applications of science and technology in different sectors of the nation and opens the door for participation in space exploration in near future.

All the above programmes can be conducted individually or in collaboration with national and international organisations working in the field of astronomy and related science. This will help the rapid development of our country.

\section{Conclusions}

The Government of Nepal should make effective plans and give significant budget for the promotion of astronomy. Especial programmes should be launched to dispel and reduce the existing superstitions in society. Organisations and individuals working in the field of astronomy and related science should be promoted. Possibilities for astro-tourism should be explored and the establishment of research centers for Gamma Ray Astronomy, Optical Astronomy and Radio Astronomy, which have great potentials in Nepal, must be done for the rapid development of our society in Nepal.

\section{Acknowledgements}

We would like to express our sincere gratitude to Er. Rishi Shah, Academician (Nepal Academy of Science and Technology, NAST), and President (Nepal Astronomical Society, NASO) for providing necessary guidance, comments and suggestions and being the source of inspiration to work in the field of astronomy and to promote astronomy for the development of our society. We are very thankful to the National Archive, the National Library and the Keiser Library for their cooperation during the preparation of this paper. 